moral, and vocational disability of a man who has lost a limb might not be as gross as popular idea would have us believe.' Great strides have been made in this branch of surgery since Vanghetti experimented with birds at Pisa and Ceci performed the first cineplastic amputation on a human being at the turn of the century. Such operations, however, should only be performed in a special unit where each new patient can be inspired by the achievements of his fellow amputees, but even under ideal conditions the mental calibre of the individual will still remain the deciding factor between success or failure.

In the past some 60 per cent. of patients have been satisfied with their cineplastic stumps and continued to use their prosthesis, but with the other 40 per cent., the limbs have eventually found their way to the lumber room and the empty sleeve pinned to the jacket remains as a silent rebuke to the surgeon. Let us hope that the advances which EyreBrook describes in his article may help to reduce the numbers of these unfortunate people.

\section{To Clot or Not to Clot}

When reading through recent publications in experimental medicine and surgery, one cannot help remarking that whilst one school is actively engaged in preventing thrombosis with the aid of heparin and dicouramin, an equally active band of workers is exploring all manner of ways in which to induce clotting, especially by the introduction of absorbable materials.

In this issue there appears a review of the new edition of Jorpes' book on 'Heparin in the Treatment of 'Thrombosis.' This publication, which has been beautifully printed in Sweden, gives a most comprehensive picture of the work on this subject carried out in Stockholm and might well serve as a model for all authors who essay to write a monograph.

In arresting haemorrhage, especially from an oozing surface, a real advance appears to have been made with the development of 'calcium alginate.'3 4 This substance was elaborated from seaweed in this country during the war, and was found to be absorbed by human tissues. When placed in contact with a bleeding surface it rapidly produces haemostasis. It has now been manufactured on a commercial basis in various forms, wool, gauze and foam being probably the most useful. It can be sterilized by autoclaving and is pleasant to handle, which makes it more acceptable than the fibrin sponge and oxycellulose preparations which have recently been evolved in the United States. Calcium alginate is slowly but completely absorbed according to the bulk of the material and it is reported to inhibit fibrosis. Only extended clinical trials can now show whether this substance will do all that is claimed for it.

\footnotetext{
${ }^{3}$ Blaine, Lancet, Oct. I 2, 1946.

'Blaine, Annals of Surgery, Jan., 1947.
} 